\title{
Questionnaires for measuring fatigue and its impact on health perception in inflammatory bowel disease
}

\author{
María Dolores Castillo-Cejas, Virginia Robles, Natalia Borruel, Antonio Torrejón, Ester Navarro, \\ Andrés Peláez and Francesc Casellas \\ Crohn-Colitis Care Unit (UACC). Gastroenterology Department. Hospital Universitari Vall d'Hebron. Barcelona, Spain
}

\begin{abstract}
Introduction: fatigue impacts perceived health, but its importance in inflammatory bowel disease is not known.

Objectives: to define the applicability of the fatigue measurement questionnaires and analyze it in patients with Crohn's disease and ulcerative colitis.

Material and methods: in a first phase, the psychometric properties of 3 fatigue measurement questionnaires were determined in 99 patients: Daily Fatigue Impact Scale, Fatigue Severity Scale, and Modified Fatigue Impact Scale. In a second phase, fatigue status and its relationship to disease and quality of life was determined in 127 patients and 69 healthy controls.

Results: the first part of the study showed the applicability of the questionnaires listed in inflammatory bowel disease, the Daily Fatigue Impact Scale (DFIS) having the best correlation with the quality of life and clinical activity. In the second phase, significantly higher levels of fatigue were observed in active disease than in disease in remission and healthy controls $(p<0,05)$. The severity of fatigue was significantly correlated with quality of life $(r=-0.66$ and -0.72 between IBDQ-9 and DFIS and in Crohn's disease and ulcerative colitis, respectively) and with disease activity $(r=0.25$ and Crohn's disease and ulcerative colitis, respectively, $p<0.05$ ).

Conclusions: in inflammatory bowel disease, fatigue measurement questionnaires have good properties and show that fatigue is an important manifestation of the disease, which has a significant impact on quality of life of patients.
\end{abstract}

Key words: Fatigue. Inflammatory bowel disease. Quality of life. Validation. Questionnaire.

Castillo-Cejas MD, Robles V, Borruel N, Torrejón A, Navarro E, Peláez A, Casellas F. Questionnaires for measuring fatigue and its impact on health perception in inflammatory bowel disease. Rev Esp Enferm Dig 2013;105:144-153.

Received: 08-10-2012

Accepted: $17-01-2013$

Correspondence: Francesc Casellas. Unitat d'Atenció Crohn-Colitis. Hospital Universitari Vall d'Hebron. Pso. Vall d'Hebron, 119. 08035 Barcelona, Spain e-mail: fcasellas@vhebron.net

\section{INTRODUCTION}

Inflammatory bowel disease (IBD) includes Crohn's disease (CD) and ulcerative colitis (UC). Both are chronic inmunoinflammatory diseases of fluctuating course that are associated with complications, including the risk of neoplastic processes (1). They require continuous treatment, with potential side effects that must be controlled and, eventually, surgery $(2,3)$. IBD limits the quality of life of patients as a result of the events inherent to it, its complications, and their treatments $(4,5)$, and also due to more subjective variables of the patient itself, affecting all domains of life (6-8).

Assessment of quality of life in relation to health has gained great importance in recent years (9-11). Given its multidimensional nature, its course over time, and its subjectivity, measuring quality of life is complex. Because of this, administration of questionnaires specifically designed for that purpose is used (12).

As in other gastrointestinal chronic intestinal diseases, such as celiac disease (13), fatigue is a manifestation reported by many patients with IBD, deeply influencing their quality of life (14) and representing a major health-determining factor (15-17).

Fatigue is the feeling of tiredness with reduced energy levels, decreased muscle strength, and cognitive impairment. As a symptom, chronic fatigue is that which persists for longer than 6 months, generally associated with a chronic illness and not related to exertion or completely improved with rest (18). It is characterized by its subjective nature and depends on many variables. Several scales have been developed for its measurement (19). However, in our setting, there is no information on the applicability of questionnaires for measuring fatigue in patients with IBD, or the impact of fatigue on quality of life in these patients.

Therefore, the objectives of this study were, on the one hand, to assess in patients with IBD the applicability of the following three questionnaires for measuring fatigue: Daily 
Fatigue Impact Scale (DFIS), Fatigue Severity Scale (FSS) and Modified Fatigue Impact Scale (MFIS), and to determine which had better application characteristics. On the other hand, to determine the impact of fatigue on health perception in patients with IBD in our setting. A prospective study was thus carried out in two phases in patients with IBD and healthy controls to whom questionnaires on fatigue and quality of life were administered.

\section{METHODS}

A prospective, observational cross-sectional study was designed in patients with $\mathrm{CD}$ and $\mathrm{UC}$, unpreselected, in two phases:

First phase, validation of fatigue measurement questionnaires: Daily Fatigue Impact Scale (DFIS), Fatigue Severity Scale (FSS), and Modified Fatigue Scale (MFIS).

Second phase, administration of the DFIS questionnaire to quantify fatigue in patients with IBD and correlate it with the degree of activity and quality of life status.

\section{Participants}

Patients included in the study were from the Crohn-Colitis Care Unit (UACC) of the Hospital Universitari Vall d'Hebron, as well as patients admitted to this hospital for a relapse of their disease. All patients, over 18 years of age, had been previously diagnosed according to standard criteria (clinical, endoscopic and histological) $(20,21)$, had different states of clinical activity and had expressed their consent to participate in the study.

Patients were stratified into 2 groups according to their diagnosis, CD versus UC. Patients with inability to complete the questionnaires, those with an underlying psychiatric illness or with other relevant chronic disease were excluded.

Additionally, a third study group consisting of healthy subjects was included. This control group was used to give meaning to the values of the fatigue questionnaire in patients from the healthy population, given the lack of data on normal values in the reference population. Inclusion criteria for the control group were the absence of disease and age between 18 and 65 years.

Disease activity in participants was measured using standard clinical indexes (22): Colitis Activity Index (CAI) for UC and Harvey-Bradshaw index for CD. Patients were considered in remission if the score was less than 6 and 3, respectively.

\section{Measurement of fatigue}

The fatigue questionnaires evaluated to determine applicability in IBD were the DFIS, FSS, and MFIS.

The DFIS is a unidimensional scale containing 8 items with 5 options each, so that the total score ranges from 0 to 32 , where a higher score indicates more severe fatigue $(23,24)$.

The FSS has been widely used in its English version and translations into other languages (including Spanish). It has been applied previously to neurological diseases, like multiple sclerosis (25), and other chronic diseases of autoimmune origin (26). The FSS is a multidimensional scale assessing psychosocial and generic aspects. It consists of 9 items with 7 possible values (where 0 indicates "totally disagree", 4 "neither agree nor disagree", and 7 is "totally agree"). The cutoff point is placed at 5 to differentiate between the presence or absence of the symptom.

The MFIS is a multidimensional scale that has been used in other chronic diseases. It has been shown that there are no differences in applying the MFIS between several languages, including Spanish, and different cultures (27). It consists of 21 items distributed into 3 subscales: physical, cognitive, and psychosocial. The final score ranges from 0 and 84 , and a score of 38 has been established as the cutoff point to define the presence or absence of fatigue.

\section{Measurement of quality of life}

For measurement of health-related quality of life, the generic EuroQol-5D questionnaire and the disease-specific IBDQ-36 and IBDQ-9 questionnaires were used.

The EuroQol-5D is a questionnaire that has been widely validated in different languages and countries, including Spanish, for which normal values have been reported for Spanish and Catalan populations $(28,29)$. It has also been assessed in a wide variety of chronic diseases (30-32) including IBD $(4,33,34)$. It evaluates 5 dimensions of health status which are: Mobility, self-care, daily life activities, pain/discomfort, and anxiety/depression $(35,36)$. Each item has 3 possible answers, where 1 is not having problems with regard to the assessed variable and 3 is having limiting problems. The result is expressed as a tariff ranging from 0 to 1 , considering 1 the best possible state and 0 the worst. The EuroQol-5D also includes a visual analog scale (VAS) for quality of life (37). The VAS consists of a $100 \mathrm{~mm}$ vertical line, like a thermometer, ranging from 0 (worst imaginable health state) to 100 (best imaginable health state). The individual should mark the point on the vertical line that best reflects, in their opinion, the assessment of their general health status on the day of data collection.

The IBDQ $(38,39)$ is a specific questionnaire for measuring quality of life in patients with IBD. There are different versions of the IBDQ duly translated and validated in Spanish. In the first phase of the study, the 36 item version (40) was used. In the second phase, the unidimensional IBDQ-9 short version with 9 questions was used (41). Each question is answered using a Likert scale from 1 to 7 , where 1 represents worst quality of life and 7 the best. The result of the IBDQ-9 is expressed by a score transformed into a percentage on a scale of $0-100$, with better health status corresponding to a higher score. 
Table I. Demographic and disease characteristics of patients included in the first phase of the study, questionnaire validation

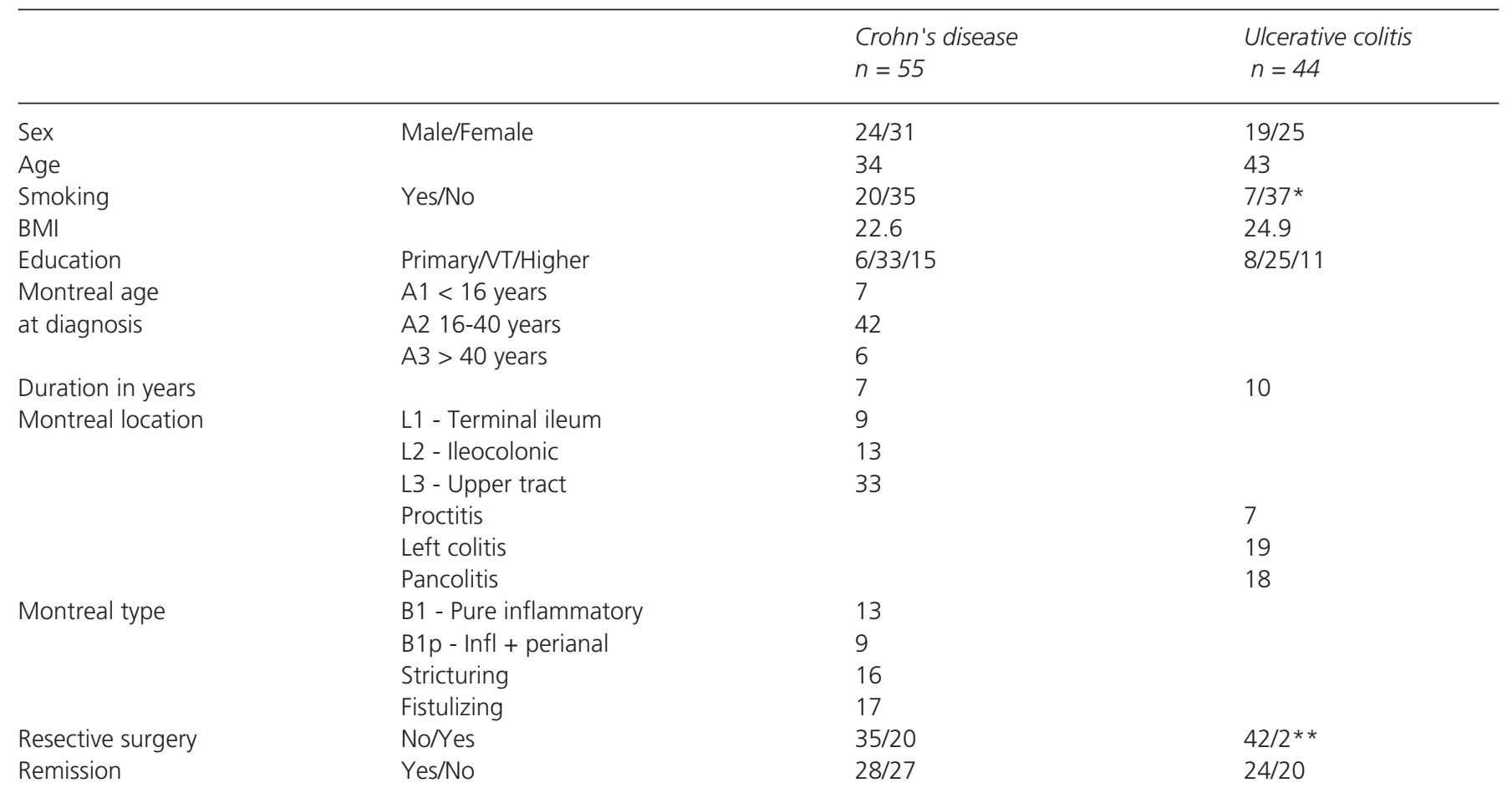

A higher percentage of smokers (36.3\%) was observed among patients with CD as well as a higher proportion of patients with prior surgery compared to patients with UC $(p<0.05) \cdot{ }^{*}=p<0.05 . * *=p<0.01$

\section{Procedure}

Participants of both phases of the study underwent a clinical evaluation related to their disease status (type, activity, manifestations, etc.) and were requested to give consent to participate in the study.

In the first phase of the study, the fatigue measurement questionnaires DFIS, FSS and MFIS and the IBDQ-36 quality of life questionnaire were administered. In the second phase, after clinical classification, the questionnaires DFIS for quantification of fatigue, IBDQ-9 and EuroQol-5D-Tariff and VAS scale were administered.

\section{Statistical analysis}

The records with the information on the included cases were managed using an Excel spreadsheet. The statistical data analyses were made using the SigmaStat version 3.5 computer program and SPSS Statistics version 17.0. Since many variables did not follow a normal distribution according to the Kolmogorov test, the results were expressed as medians and percentiles [25-75], and the statistics used were based on nonparametric tests. Quantitative variables were compared using the Mann-Whitney or KruskalWallis statistical tests as required for unpaired samples. A value of $p<0.05$ was considered statistically significant.

\section{RESULTS}

\section{Questionnaire validation phase}

A total of 99 patients were included, 55 with $\mathrm{CD}$ and 44 with UC. Both groups had a similar distribution in terms of sex, age, and time since diagnosis. The only differences were related to smoking and the number of prior surgeries (Table I). It was observed that the group of CD patients had a higher percentage of smokers $(p<0.05)$ as well as a higher proportion of patients with prior surgery compared to UC patients $(\mathrm{p}<0.01)$.

The correlation was then established between the three fatigue questionnaires (Fig. 1), all showing a positive correlation, with values " $r$ " between 0.77 and 0.84 , with $\mathrm{p}<$ 0.001 in the three cases.

Next, the correlation was established between fatigue measured using the three questionnaires and quality of life measured with the global score of the IBDQ-36. It was observed that the results of the three fatigue questionnaires had an inverse relationship, showing that the greater the fatigue, the poorer the quality of life perceived. A statistically significant correlation of IBDQ-36 with the three fatigue questionnaires was obtained (with a $\mathrm{r}$ between -0.72 and $-0.81, p<0.001$ ), and the correlation with the DFIS was slightly higher than with the other two questionnaires (Fig. 2). 


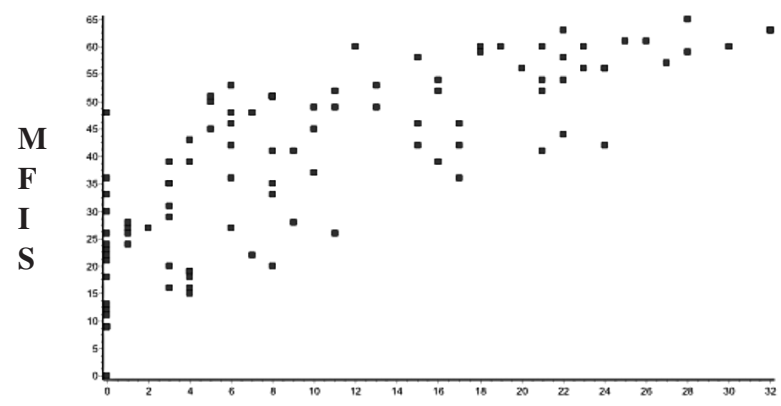

F S S

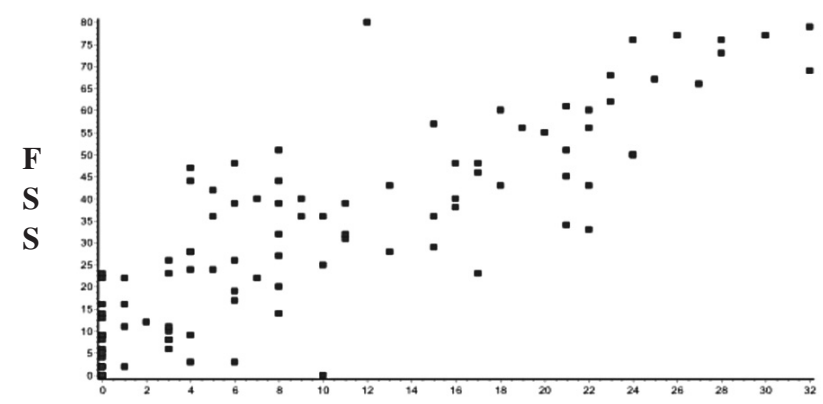

D F I S

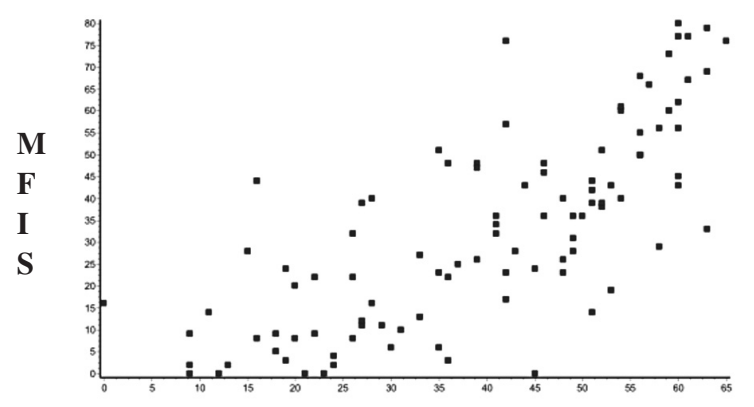

D F I S

Fig. 1. Correlation between the three fatigue questionnaires. Each point corresponds to the individual score obtained by each patient. A. Between the MFIS and FSS, $r=0.77$ was obtained with $p<0.001$, which was statistically significant. B. Between the FSS and DFIS, $r=0.82$ was obtained with $p<$ 0.001 , and was statistically significant. C. Between the MFIS and DFIS, $r=0.84$ was obtained with $p<0.001$, and was statistically significant (MFIS: Modified Fatigue Impact Scale; FSS. Fatigue Severity Scale; DFIS: Daily Fatigue Impact Scale).
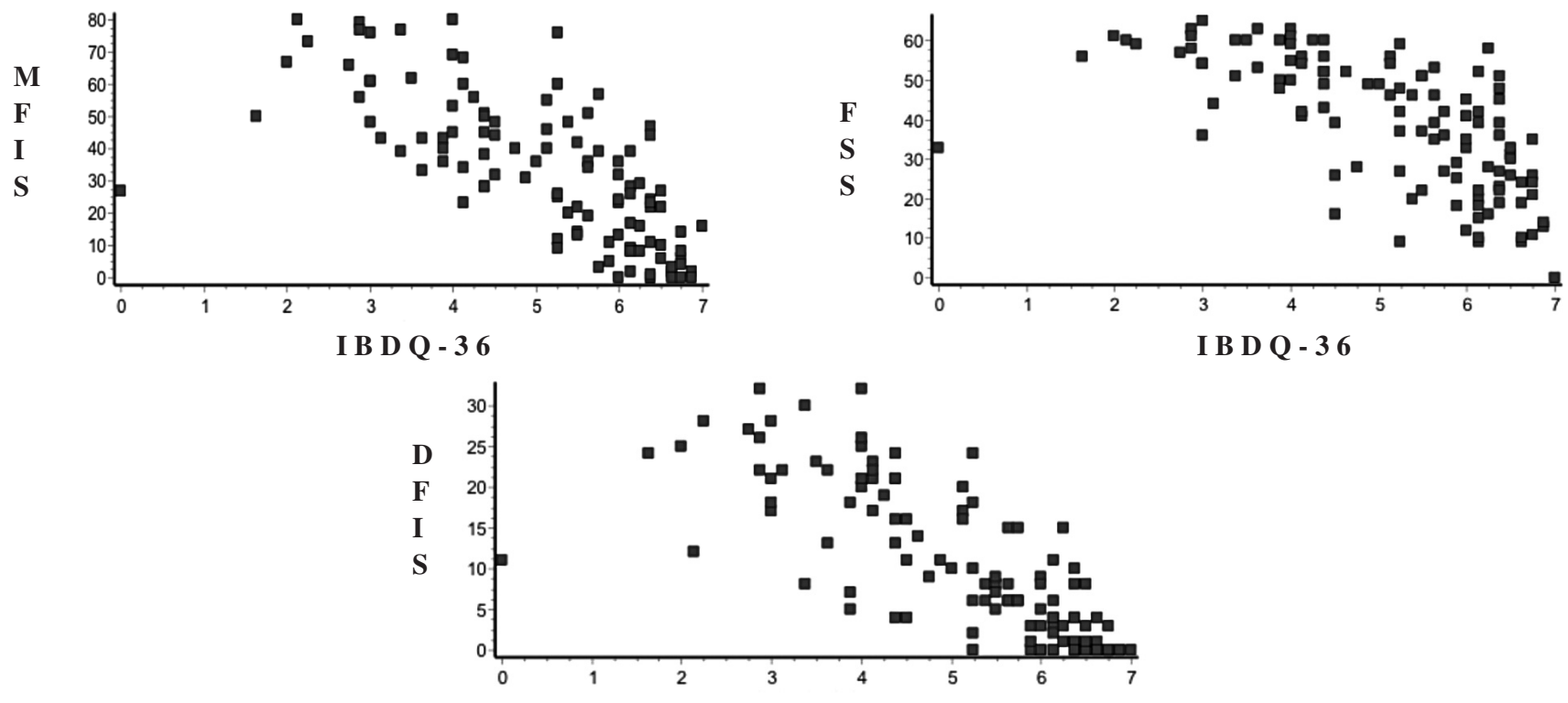

I B D Q - 36

Fig. 2. Correlation between the three fatigue questionnaires and the IBDQ-36. Each point corresponds to the individual score obtained by each patient. Inverse correlations were obtained, with poorer quality of life with greater fatigue, which was statistically significant between the IBDQ-36 and the three fatigue scales. A. With the MFIS, $r=-0.76$ was obtained with $p<0.001$. B. With the FSS, $r=-0.72$ was obtained with $p<0.001$. C. With the DFIS, $r=0.81$ was obtained with $p<0.001$ (MFIS: Modified Fatigue Impact Scale; FSS: Fatigue Severity Scale; DFIS: Daily Fatigue Impact Scale; IBDQ: Inflammatory Bowel Disease Questionnaire). 
The relationship between fatigue and clinical activity of the disease was established by correlation of the three questionnaires (DFIS, FSS, and MFIS) with the score on the Harvey-Bradshaw index in patients with CD and the score on the CAI in patients with UC. The results obtained showed a positive relationship between the degree of disease activity and the level of fatigue in the three questionnaires tested, with the greater the fatigue the greater the clinical activity. The questionnaire with greatest potency in this relationship, both in UC and CD patients, was the DFIS. It obtained $r=0.67(p<0.001)$ for CD and $r=0.50$ $(\mathrm{p}<0.001)$ for UC with the FSS. These values were $r=0.53(p<0.001)$ for CD and $r=0.37(p<0.05)$ for $U C$; with the MFIS, the values were $r=0.48(p<0.001)$ for CD and $r=0.39(p<0.01)$ for UC.

Based on the results described above, we consider that the three fatigue measurement questionnaires tested in IBD were valid, but the DFIS was chosen for the next phase of the study because it showed the greatest correlation with clinical activity and quality of life of patients.

\section{Questionnaire administration phase}

A total of 137 patients were included in this phase, 70 with a diagnosis of $\mathrm{CD}$, and 67 with a diagnosis of $\mathrm{UC}$, different from the validation phase. Overall data are shown in Table II, suggesting that UC patients had lower tobacco consumption and a higher median age than CD patients, reaching a $p$ value with statistical significance. Another difference was that there was a higher percentage of smokers among patients with CD. Regarding clinical characteristics, $\mathrm{CD}$ patients had been diagnosed younger than the UC patients, and had required a higher percentage of surgery, $50 \%$ in CD patients versus $3 \%$ in UC patients $(p<0.05)$. It was also found that there was a higher percentage of patients in relapse in the CD group (24.3\%) than in the UC group. At the time of data collection, a follow-up laboratory test was obtained to determine the presence of anemia, vitamin B12 deficiency and acute phase reactants. These parameters were also analyzed according to whether patients were in relapse or remission, revealing (Table III) that patients in relapse had elevated acute phase reactants (leukocyte count, platelet count, and CRP, $p<0.001$ ) to a greater extent than patients in remission. Moreover, they had lower hemoglobin values (median $13.3 \mathrm{~g} / \mathrm{dl}$ in patients in remission and $12.6 \mathrm{~g} / \mathrm{dl}$ in patients in relapse) $(\mathrm{p}<0.05)$, with no differences between the CD group and UC group.

Measurement of fatigue was performed according to the DFIS questionnaire and quality of life according to the EuroQol-5D and IBDQ-9 questionnaires. No statistically significant differences were observed between CD and UC patients in scores on the three questionnaires. However, there was a certain trend to score worse on the DFIS in patients with CD than in those with UC (median $9.5 \mathrm{vs}$. 6.0 , respectively), although without reaching statistical significance.
Analysis was then performed by stratifying patients according to the degree of disease activity. It was found that patients in relapse scored worse on the quality of life questionnaires, both IBDQ-9 and EuroQol-5D, and had a greater fatigue index on the DFIS questionnaire, with no differences between CD and UC (Table IV).

The relationship between clinical disease activity and level of fatigue, on the one hand, and quality of life and level of fatigue on the other, was statistically significant in both cases (Fig. 3). The correlation of clinical activity with fatigue was $r=0.34$ and $r=0.26$ in $\mathrm{UC}$ and $\mathrm{CD}$, respectively $(\mathrm{p}<0.01)$. Similarly, the correlation of clinical activity with quality of life was also statistically significant in $\mathrm{UC}$ and $\mathrm{CD}(\mathrm{r}=-0.62$ and $\mathrm{r}=-0.47$, respectively, $\mathrm{p}<0.001$ (Fig. 4). Also, when establishing the correlation between the degree of fatigue and quality of life, values of inverse correlation were obtained reaching statistical significance $(\mathrm{r}=-0.73$ and $\mathrm{r}=-0.67$ for $\mathrm{UC}$ and $\mathrm{CD}$, respectively, $\mathrm{p}<$ 0.001) (Fig. 5).

As mentioned above, given the lack of normal ranges in our population, a control group of healthy individuals was established to determine the degree of perception of fatigue in people without disease. A total of 69 people were included. The demographic data are shown in Table II, with no statistically significant differences versus the group of patients.

Comparison of the DFIS score in the group of healthy individuals versus the group of patients, overall and by patients in relapse or in remission, showed that healthy controls had a lower level of fatigue compared to patients (4.0 vs. 9.0, respectively, $\mathrm{p}<0.01$ ). According to the stratification of patients by disease activity, a poorer DFIS score was seen in both groups of patients compared to controls (Table V).

\section{DISCUSSION}

Little information is available on the impact of fatigue or its impact on quality of life in patients with IBD. Therefore this study was designed in two sequential stages with two concrete objectives. The first objective was to establish whether fatigue measurement questionnaires available in Spanish -DFIS, FSS, and MFIS- were applicable to patients with IBD. The second objective of the study was to analyze fatigue in IBD by administering the questionnaire with the best properties, and determining its influence on the quality of life of patients. Lastly, the importance of fatigue in IBD was established by comparing the results obtained in patients with those of a control group of healthy subjects.

The results obtained in the study show that the three fatigue measurement questionnaires analyzed have adequate properties for use in IBD, with the DFIS standing out as the one which achieves better correlations with clinical activity and quality of life of the patients. Our results also suggest that fatigue is a major problem in IBD, both in CD 
Table II. Demographic and disease characteristics of controls and patients included in the analysis of fatigue and its impact on the quality of life

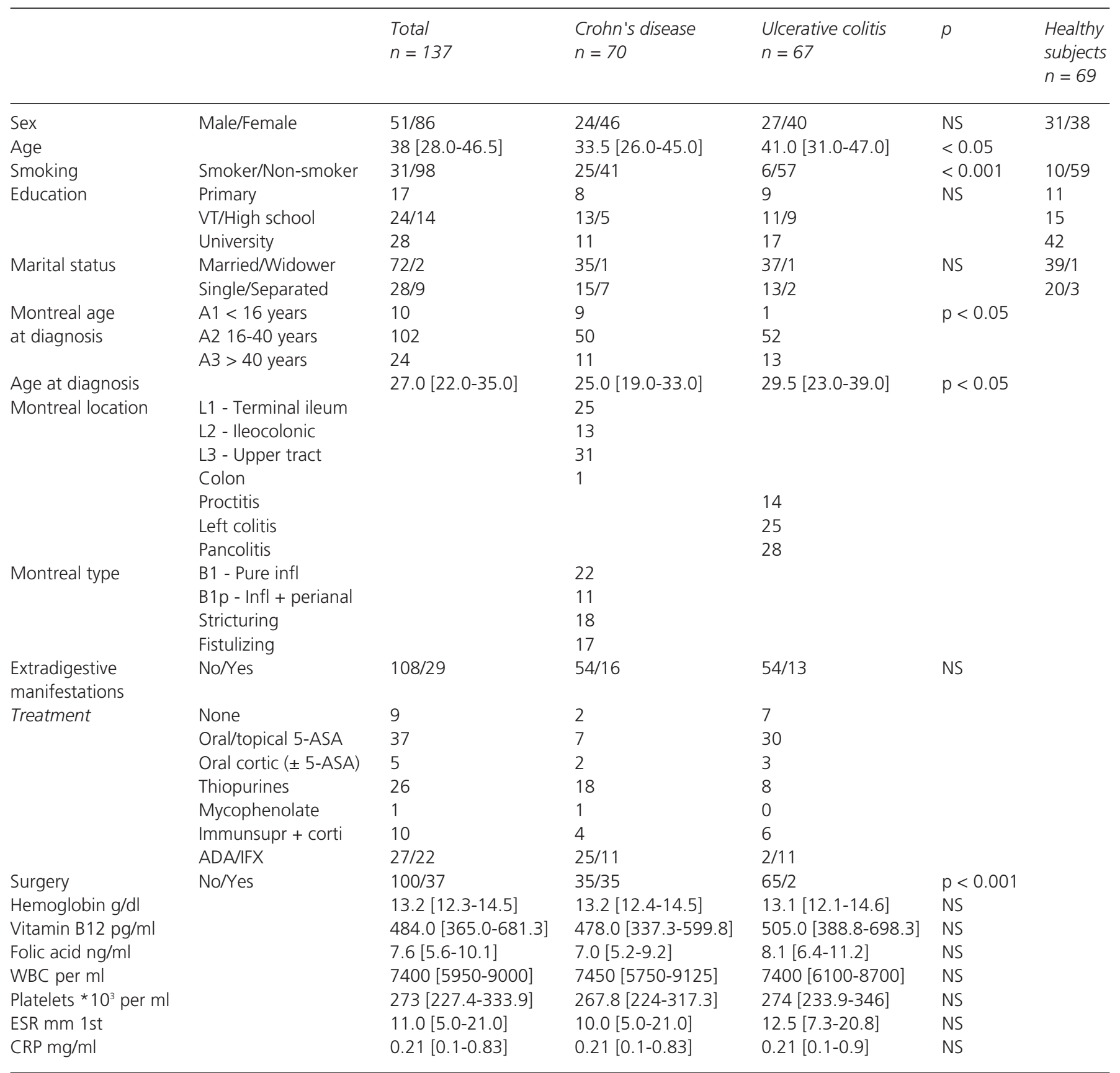

and UC, and is influenced by various factors, particularly disease activity. In addition, fatigue impacts unfavorably on patient quality of life, in both CD and UC, so that greater fatigue is related to poorer quality of life.

Both CD and UC patient groups had similar demographic characteristics, as well as a similar disease course, except for the greater need for surgery in patients with CD. Laboratory parameters of both groups also showed significant differences, except for higher acute phase reactants in patients in relapse (CD or UC) than in those in remission.
Previously, the study by Jelsness et al. (42) had shown in a Norwegian population that the greater the fatigue, the poorer IBD patients scored on quality of life tests, both in $\mathrm{CD}$ and UC, particularly in the domains of physical function, which caused them to have problems to comply with their work/studies. They also found that having chronic fatigue as a symptom caused patients to have a greater degree of concern about their disease, which in turn led to poorer, perceived quality of life. In our study, a similar score was found in the quality of life questionnaires between CD 
Table III. Distribution of laboratory parameters in patients according to disease activity

\begin{tabular}{llll}
\hline & Remission & Relapse & $p$ \\
\hline $\mathrm{Hb} \mathrm{g} / \mathrm{dl}$ & $13.3(12.4-14.6)$ & $12.6(10.7-13.4)$ & $<0.05$ \\
Vitamin B12 pg/ml & $488.5(366-682)$ & $455.5(297.25-662)$ & $\mathrm{NS}$ \\
Folic acid $\mathrm{ng} / \mathrm{ml}$ & $7.74(6.05-10.55)$ & $6.25(4.2-8.45)$ & $<0.05$ \\
WBC per ml & $7300(5600-8800)$ & $8700(6800-11500)$ & $<0.05$ \\
Platelets per ml & $263200(222375-328900)$ & $303300(274000-428800)$ & $<0.01$ \\
ESR mm 1st $\mathrm{h}$ & $11(6-19.75)$ & $19(5-44)$ & $\mathrm{NS}$ \\
CRP mg/ml & $0.2(0.073-0.50)$ & $1.49(0.19-3.61)$ & $<0.001$ \\
\hline
\end{tabular}

No statistically significant differences were observed between CD and UC groups, but there were differences between patients in relapse or remission. (Hb: Hemoglobin; WBC: white blood cell counts; ESR: erythrocyte sedimentation rate; CRP: C-reactive protein).

Table IV. Patient scores on questionnaires according to disease activity and CD or UC

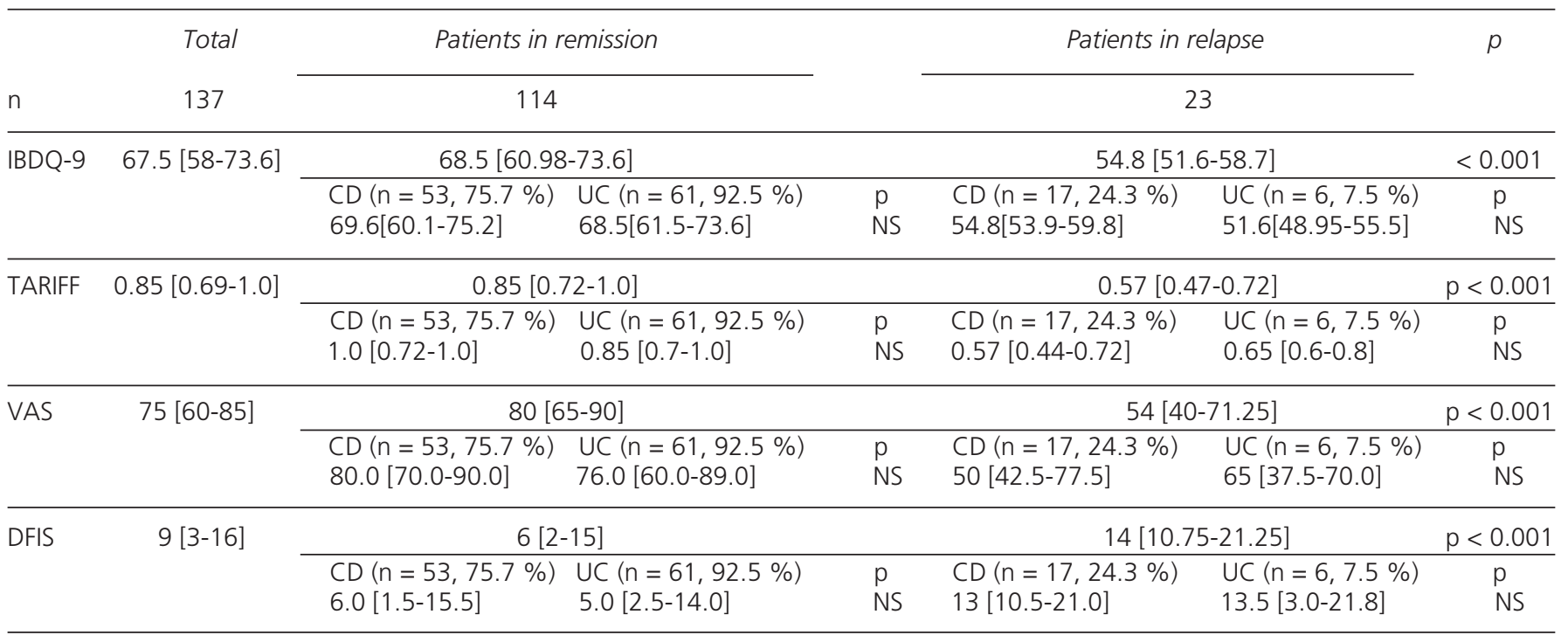

It was found that patients in relapse showed poorer quality of life and a greater level of fatigue compared to patients in remission, with the difference reaching statistical significance. When subdivided by CD or UC, statistically significant differences were not found between the two groups. (CD: Crohn's disease; UC: ulcerative colitis; IBDQ: Inflammatory Bowel Disease Questionnaire; DFIS: Daily Fatigue Impact Scale; VAS; visual analog scale).

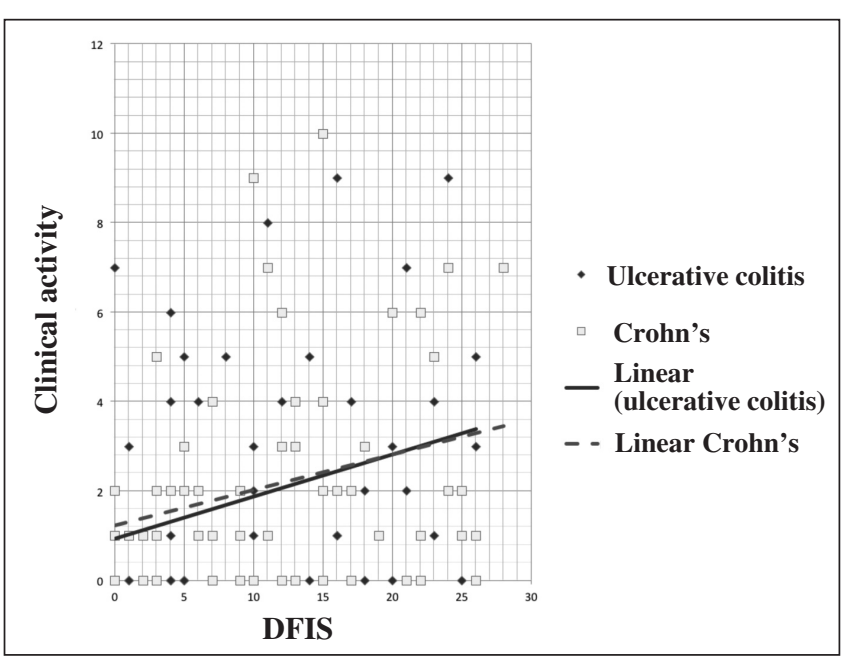

Fig. 3. Correlation between clinical activity and score on DFIS. There was a positive correlation between the two parameters in both UC and CD patients. In the case of UC patients, the index of relation was $r=0.3399$ with $p<0.005$, statistically significant. In CD patients, the index was $r=0.2590$ with $p<0.05$, also statistically significant (CD: Crohn's disease; UC: Ulcerative colitis; DFIS: Daily Fatigue Impact Scale).

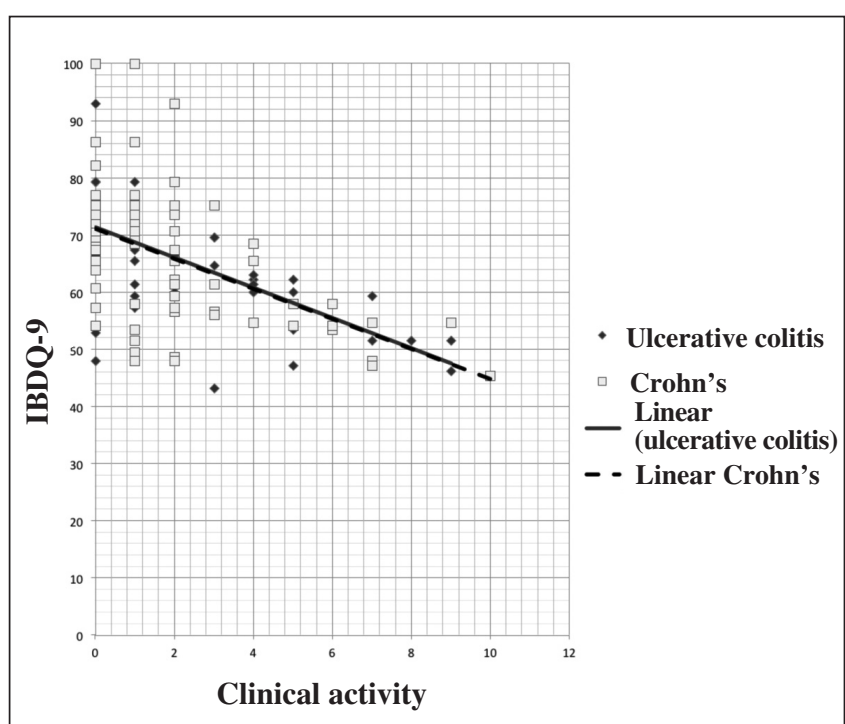

Fig. 4. Correlation between clinical activity and perception of quality of life. Inverse correlations were observed in both patient groups, with $r=-0.6254$ with $p<0.001$ in the UC group, and $r=-0.4736$ with $p<$ 0.001 in the CD group (CD: Crohn's disease; UC: Ulcerative colitis). 


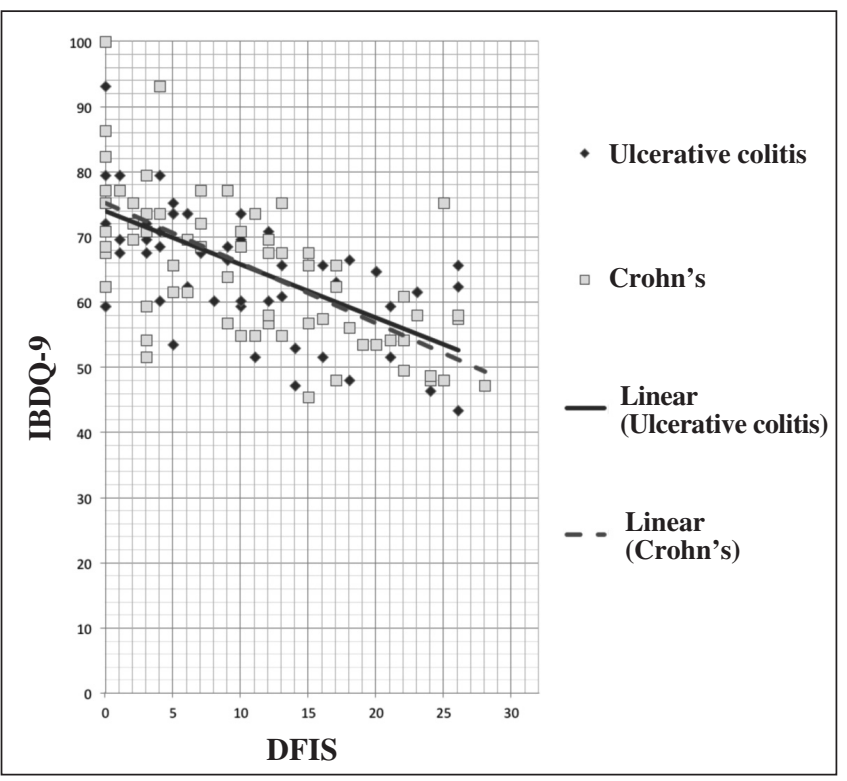

Fig. 5. Correlation between perception of quality of life and score on DFIS. There was a positive correlation between the two parameters in both UC and CD patients. In the case of UC patients, the index of relation was $r=0.3399$ with $p<0.005$, statistically significant. CD patients also showed an inverse relationship with $r=-0.6671$ with $p<0.001$, statistically significant (DFIS: Daily Fatigue Impact Scale; CD. Crohn's disease; UC: Ulcerative colitis).

and UC groups, measured both with generic instruments and specific for IBD. When fatigue was quantified, no statistically significant differences were observed between both groups, although a clear trend was shown to score worse, that is, express greater fatigue, in the CD group. This could be related to the higher percentage of patients in relapse of the disease in the group of CD patients compared to the percentage of patients in relapse in the group of UC patients. Quality of life and the fatigue index showed an inversely proportional relationship, with a statistically significant index of relation demonstrating that more fatigue is associated with poorer quality of life. The Kruskal-Wallis statistical analysis confirmed fatigue as a determinant of poor quality of life, which was observed in both $\mathrm{CD}$ and UC.

Another of our objectives in the study was to establish whether the disease activity index is related to fatigue. Römkens et al. (43) evaluated a group of Dutch patients with IBD and showed that patients reporting more fatigue were those with greatest activity. Our patients were stratified between high activity index (considered as relapse) and low activity index (considered as remission) and poorer perceived quality of life was found in patients in relapse with statistically significant differences, both in patients with CD and UC. When the relationship was established between quality of life and clinical disease activity, an inverse trend was seen between both parameters so that the greater the activity, the poorer perceived quality of life, with statistical significance. This same relationship was
Table V. Comparison of DFIS score between the healthy subject group and the patient group

\begin{tabular}{llll}
\hline & Healthy subjects & Patients & $p$ \\
\hline$n$ & 69 & 137 & \\
\hline DFIS & $4.0[1.0-8.5]$ & $9.0[3.0-16.0]$ & $<0.005$ \\
& & Remission $(n=114)$ & \\
& $6.0[2.0-15.0]$ & $<0.05$ \\
& & Relapse $(n=23)$ & \\
& $13.0[10.8-21.8]$ & $<0.001$
\end{tabular}

Healthy subjects had a lower level of fatigue compared to patients, whether they were in relapse or remission. The control group had a median DFIS score of 4 , while patients scored 9 (patients in remission scored 6 and patients in relapse 13), and when comparisons were made, they acquired statistical significance. DFIS: Daily Fatigue Impact Scale. n: Number of patients.

also found between the activity index and patient fatigue, in such a way that patients with a greater activity index expressed more fatigue. In this study, the influence of other factors on fatigue was not determined, an aspect that remains open for future projects.

In our study, the fatigue index obtained in patients with IBD was compared with a healthy control group, and the patients in general had a greater DFIS index than the healthy subjects, which is in line with the results of the study conducted by Römkens et al. (43). On stratifying patients in relapse and remission, it was seen that patients in remission also scored poorer than healthy subjects, with the difference reaching statistical significance.

This study has the limitations of the small number of patients included, which prevented performing subanalyses of groups to compare patients according to their extradigestive manifestations, extension of disease and other related aspects. This study could be expanded in the future, which would also allow for other factors to be established which influence the perception of fatigue by patients regardless of the underlying disease.

In conclusion, from this study it can be stated that in inflammatory bowel disease, both CD and UC, fatigue measurement questionnaires, particularly the DFIS, have properties which make their use appropriate. It has also been confirmed in our setting that fatigue is an important manifestation of the disease, particularly in relapse, and significantly impacts the quality of life of patients.

\section{REFERENCES}

1. Nugent FW, Haggitt RC, Gilpin P. Cancer surveillance in ulcerative colitis. Gastroenterology 1991;100:1241-8.

2. Taxonera C, Rodrigo L, Casellas F, Calvet X, Gómez-Camacho F, Ginard D, et al. Infliximab maintenance therapy is associated with decrease in direct resource use in patients with luminal or fistulizing Crohn's disease. J Clin Gastroenterol 2009;43:950-6.

3. Mowat C, Cole A, Windsor A, Ahmad T, Arnott I, Driscoll R, et al. Guidelines for the management of inflammatory bowel disease in adults. Gut 2011;60:571-607. 
4. Casellas F, López Vivancos J, Sampedro M, Malagelada JR. Relevance of the phenotypic characteristics of Crohn's disease in patient perception of health-related quality of life. Am J Gastroenterol 2005;100:2737-42.

5. Casellas F, Arenas JI, Baudet JS, Fabregas S, Garcia N, Gelabert J, et al. Impairment of health-related quality of life in patients with inflammatory bowel disease: A Spanish multicenter study. Inflamm Bowel Dis 2005;11:488-96.

6. Casellas F, López-Vivancos J, Casado A, Malagelada JR. Factors affecting health related quality of life of patients with inflammatory bowel disease. Qual Life Res 2002;11:775-81.

7. Vergara M, Montserrat A, Casellas F, Gallardo O, Suarez D, Motos J, et al. Development and validation of the Crohn's disease perceived work disability questionnaire. Inflamm Bowel Dis 2011;17:2350-7.

8. Casellas F, López-Vivancos J, Badia X, Vilaseca J, Malagelada JR. Influence of inflammatory bowel disease on different dimensions of quality of life. Eur J Gastroenterol Hepatol 2001;13:567-72.

9. Velarde-Jurado E, Ávila-Figueroa C. Evaluación de la calidad de vida. Salud Pública Mex 2002;44:349-61.

10. Guyatt GH, Feeny DH, Patrick DL. Measuring health-related quality of life. Ann Intern Med 1993;118:622-9.

11. Testa MA, Simonson DC. Assessment of quality-of-life outcomes. N Engl J Med 1996;334:835-40.

12. Lock K. Health impact assessment. BMJ 2000;320:1395-8.

13. Casellas F, Rodrigo L, López Vivancos J, Riestra S, Pantiga C, Baudet JS, et al. Factors that impact health-related quality of life in adults with celiac disease: A multicenter study. World J Gastroenterol 2008;14:46-52.

14. Jelsness-Jørgensen LP, Bernklev T, Henriksen M Torp R, Moum BA. Chronic fatigue is more prevalent in patients with inflammatory bowel disease than in healthy controls. Inflamm Bow Dis 2011;17:1564-72.

15. Graff LA, Vincent N, Walker JR, Clara I, Carr R, Ediger J, et al. A population-based study of fatigue and sleep difficulties in inflammatory bowel disease. Inflamm Bow Dis 2011;17:1882-9.

16. Jelsness-Jørgensen LP, Bernklev T, Henriksen M Torp R, Moum BA. Chronic fatigue is associated with impaired health-related quality of life in inflammatory bowel disease. Aliment Pharmacol Ther 2011; 33:106-14.

17. Langenberg DR, Gibson PR. Systematic review: fatigue in inflammatory bowel disease. Aliment Pharmacol Ther 2010;32:131-43.

18. Swain MG. Fatigue in chronic disease. Clin Sci 2000;99:1-8.

19. Lee KA, Hicks G, Nino-Murcia G. Validity and reliability of a scale to assess fatigue. Psychiatry Res 1991;36:291-8.

20. Stange EF, Travis SPL, Vermeire S, Reinisch W, Geboes K, Barakauskiene $\mathrm{A}$, et al. European evidence-based consensus on the management of ulcerative colitis: definitions and diagnosis. J Crohns Colitis 2008:2:1-23.

21. Sands BE. From symptom to diagnosis: clinical distinctions among various forms of intestinal inflammation. Gastroenterology 2004; 126:1518-32.

22. Satsangi J, Silverberg MS, Vermeire S, Colombel JF. The Montreal classification of inflammatory bowel disease: controversies, consensus and implications. Gut 2006;55:749-53.

23. Kos D, Kerckhofs E, Carrea I, Verza R, Ramos M, Jansa J. Evaluation of the Modified Fatigue Impact Scale in four different European countries. Mult Scler 2005;11:76-80.

24. Jones DE, Gray JC, Newton J. Perceived fatigue is comparable between different disease groups. QJM 2009;102:617-24.

25. Valko PO, Bassetti CL, Bloch KE. Validation of the Fatigue Severity Scale in a Swiss cohort. Sleep 2008;31:1601-7.

26. Krupp Lb, LaRocca Ng, Muir-Nash J, Steinberg AD. The Fatigue Severity Scale. Application to patients with multiple sclerosis and systemic lupus erythematosus. Arch Neurol 1989;46:1121-3.
27. Kos D, Kerckohfs E, Carrea I, Verza R, Ramos M, Jansa J. Evaluation of the Modified Fatigue Impact Scale in four different European countries. Mult Scler 2005;11:76-80.

28. Badia X, Roset M, Herdman, Kind P. A comparison of United Kingdom and Spanish general population time trade-off values for EQ-5D health states. Med Decis Making 2001;21:7-16.

29. Badia X, Schiaffino A, Alonso J, Herdman M. Using the EuroQol 5$\mathrm{D}$ in the Catalan general population: feasibility and construct validity. Qual Life Res 1998;7:311-22.

30. Höfer S, Kullich W, Graninger U, Wonisch M, Gassner A, Klicpera $\mathrm{M}$, et al. Cardiac rehabilitation in Austria: long term health-related quality of life outcomes. Health Qual Life Outcomes 2009;7:99.

31. Harrison MJ, Lunt M, Verstappen SM, Watson KD, Bansback NJ, Symmons DP. Exploring the validity of estimating EQ-5D and SF-6D utility values from the health assessment questionnaire in patients with inflammatory arthritis. Health Qual Life Outcomes 2010;8:21.

32. Pérez-San Gregorio MA, Martín-Rodríguez A, Pérez-Bernal J, Maldonado MD. Quality of Life in Spanish patients with liver transplant. Clin Pract Epidemiol Ment Health 2010;6:79-85.

33. Huamán JW, Casellas F, Borruel N, Peláez A, Torrejón A, Castells I, et al. Cutoff values of the inflammatory bowel disease questionnaire to predict a normal health related quality of life. J Crohns Colitis 2010;4:637-41

34. Janssen MF, Birnie E, Bonsel GJ. Quantification of the level descriptors for the standard EQ-5D three-level system and a five-level version according to two methods. Qual Life Res 2008;17:463-73.

35. Craig BM, Busschbach JJV, Salomon JA. Modeling ranking, time tradeoff and visual analogue scale values for EQ-5D health states: A review and comparison of methods. Med Care 2009;47:634-41.

36. Masachs M, Casellas F, Borruel N, Torrejón A, Castells I, Malagelada JR. Validation of the Spanish version of a questionnaire to measure quality of care through the eyes of patients with inflammatory bowel disease (QUOTE-IBD). Inflamm Bowel Dis 2010;16:982-92.

37. Montejo AL, Correas-Lauffer J, Mauriño J, Villa G, Rebollo P, Díez $\mathrm{T}$, et al. Estimation of a multiattribute utility function for the Spanish Version of the TooL Questionnaire. Value Health 2011;14:564-70.

38. Masachs M, Casellas F, Malagelada JR. Spanish translation, adaptation and validation of the 32 -item quality of life questionnaire (IBDQ32) for inflammatory bowel disease. Rev Esp Enferm Dig 2007; 99:511-9.

39. Bernklev T, Jahnsen J, Lygren I, Henriksen M, Vatn M, Moum B. Health-related quality of life in patients with inflammatory bowel disease measured with the short form-36: psychometric assessments and a comparison with general population norms. Inflamm Bowel Dis 2005;11:909-18.

40. López-Vivancos J, Casellas F, Badia X, Vilaseca J, Malagelada JR. Validation of the spanish version of the inflammatory bowel disease questionnaire on ulcerative colitis and Crohn's disease. Digestion 1999;60:274-80.

41. Alcalá MJ, Casellas F, Fontanet G, Prieto L, Malagelada JR. Shortened questionnaire on quality of life for inflammatory bowel disease. Inflamm Bowel Dis 2004;10:383-91.

42. Jelsness-JØrgensen LP, Bernklev T, Henriksen M, Torp R, Moum B. Chronic fatigue is associated with increased disease-related worries and concerns in inflammatory bowel disease. World J Gastroenterol 2012;18:445-52.

43. Römkens TE, van Vugt-van Pinxteren MW, Nagengast FM, van Oijen MG, de Jong DJ. High prevalence of fatigue in inflammatory bowel disease: A case control study. J Crohns Colitis 2011;5:332-7. 